\title{
Simultaneous effect of disease and toxicant on a plant population: a model
}

\author{
Sudipa Chauhan ${ }^{1 *}$, Om Prakash Misra ${ }^{2}$, Joydip Dhar ${ }^{3}$ \\ ${ }^{1}$ Amity University, Sector-125, Noida, India \\ 2 School of Mathematics and Allied Sciences, Jiwaji University, Gwalior, India \\ ${ }^{3}$ ABV-IIITM, Gwalior, India \\ *Corresponding author E-mail: sudipachauhan@gmail.com
}

\begin{abstract}
In this paper, we have studied the effect of disease separately on the plant population and the simultaneous effect of disease and toxicant on plant population. The disease is caused to the plants by vector population and toxicants are effecting the total plant density directly through air. The local and global stability conditions for both the models have been derived and numerical simulation is done using MATLAB software.
\end{abstract}

Keywords: Plant population, Vector population, Disease, Toxicant, Stability.

\section{Introduction}

The rapid development of modern industry and agriculture have polluted our environment and ecosystem to a large extent. As a result of the pollution, the plant biomass is being adversely affected causing the reduction in the producer species utilized by the primary consumers such as herbivores. In the early eighties, a deterministic modelling approach to the problem of assessing the effects of a pollutant on an ecological system was proposed by Hallam and his co-workers [1, 2, 3, 4, 5]. Population-toxicant coupling has been applied in several contexts, including Lotka-Volterra and chemostat like environments resulting in ordinary, integro-differential and stochastic models. Usually a qualitative analysis was performed which focusses on the survival or extinction of populations [6,7]. Another important adverse factor which affects the growth of the plant population is the occurrence of plant diseases. In the case of plant diseases caused by vectors, a three way interaction exist between virus, host plant and vector. The virus is transmitted by two processes, first by acquisition of virus from infected plants and secondly by inoculation of healthy host plants by vectors. However, mathematical models of plant-virus interaction have been studied by several researchers $[7,8,9,10,11,12,13,14]$. All models of plant-virus disease that incorporate both host and vector take into account the contact rates between healthy plants and infective vectors. These are specified both by virus acquisition rate and inoculation rate, respectively. Thus, it is observed that the growth of plant population is being adversely affected both by viral infection and also by the presence of pollutant in the habitat. Therefore to visualize the simultaneous effects of pollutants and disease on the growth of plant biomass, a comprehensive mathematical model is suggested in this paper which has been analyzed using stability theory and the numerical simulation is done using MATLAB software.

\section{The mathematical formulation}

The vector population dynamics can be modelled as

$$
\frac{d W}{d t}=b W-c W
$$


where $\mathrm{b}$ is the birth rate of the vector, $\mathrm{c}$ is the loss rate of the vector due to death and emigration and $\mathrm{W}$ is the vector density per unit ground area. But this model cannot explain the abundance of the vector within a crop because immigration can occur throughout the whole crop growth period and emigration rate increases as the plant ages. Thus, a modified model of vector population dynamics of a single crop is

$$
\frac{d W}{d t}=b W-c W+\tau
$$

where $\tau$ represent a constant vector immigration rate. The plant-virus disease that incorporate both host and vector are based on contact rates, both between infectious plants and non-infective vectors and between healthy plants and infective vectors that specify virus acquisition rate by the vector and inoculation rate of the host plant given by the mathematical forms $\lambda_{2} S H$ and $\lambda_{1} Z H$ where $\lambda_{2}$ is the acquisition rate, $\lambda_{1}$ is the inoculation rate and $H, S, X$ and $Z$ are the healthy hosts, infective hosts, noninfective vectors and infective vectors, respectively. Accordingly, a model 1(a) can be formulated as:

$\frac{d H}{d t}=r(K-H)-k_{1} Z H$

$\frac{d S}{d t}=k_{1} Z H-\left(k_{3}+r\right) S$

$\frac{d X}{d t}=b(X+Z)-c X-k_{2} S X+(1-q) \tau$

$\frac{d Z}{d t}=k_{2} S X-c Z+q \tau$

where $r$ is the host mortality rate, $q$ is the proportion of imigrating vectors that are virulinferous, $K$ is the plant density. Now, let $P(t)$ is the concentration of toxicant in the environment at time $\mathrm{t}$, and $U(t)$ is the concentration of toxicant in the organism at timt $t$. Then the system (1-4) under the effect of toxicant can be given by the following system of ordinary differential equations $1(\mathrm{~b})$ :

$\frac{d H}{d t}=r(K-H)-k_{1} Z H-\alpha_{1} H U$

$\frac{d S}{d t}=k_{1} Z H-\left(k_{3}+r\right) S-\alpha_{2} S U$

$\frac{d X}{d t}=b(X+Z)-c X-k_{2} S X+(1-q) \tau$

$\frac{d Z}{d t}=k_{2} S X-c Z+q \tau$

$\frac{d P}{d t}=Q-h P$

$\frac{d U}{d t}=a_{1} P+\frac{d_{1} \eta \phi}{a_{1}}-\left(l_{1}+l_{2}\right) U$

The first two terms on the right hand side in equation (10) denote the organismal net uptake of toxicant from the environment and the food chain, respectively; due to metabolic processing and other causes. The parameters $a_{1}, d_{1}, \phi, l_{1}, \eta$ and $l_{2}$ are positive constants. $a_{1}$ denotes the environmental toxicant uptake rate per unit mass organism, $d_{1}$ denotes the uptake rate of the toxicant in food per unit mass of organism, $\eta$ is the concentration of the toxicant in thr resource, $\phi$, the average rate of food intake per unit mass organism, $l_{1}$ and $l_{2}$ are organismal net ingestion and depuration rates of toxicant respectively. The positive constant $h$ in (10) represents the loss rate of toxicant from the environment including processes such as biological transformation, chemical hydolysis, volatization, microbial degradation and photosynthesis degradation. The exogenous rate of toxicant input into the environment is represented by $Q$. System (1-4) can be shown to be mathematically well posed in the positive invariant region;

$$
R_{1}=\left\{(H, S, X, Z) \mid 0 \leq H+S \leq K, 0 \leq X+Z \leq \frac{\tau}{c-b}\right\}
$$

System (5-10) can be shown to be mathematically well posed in the positive invariant region;

$$
R_{2}=\left\{(H, S, X, Z, P, U) \mid 0 \leq H+S \leq K, 0 \leq X+Z \leq \frac{\tau}{c-b}, 0 \leq P \leq P^{*}, 0 \leq U \leq U^{*}\right\}
$$

where $P^{*}=\frac{Q}{h}, U^{*}=\frac{a_{1} P^{*}+\left(d_{1} \beta \phi / a_{1}\right)}{\left(l_{1}+l_{2}\right)}$. 


\section{Equilibria and their local stability}

In this section we will discuss the local stability behavior of the feasible boundary equilibrium point and interior equilibrium point of both the mathematical models (1-4) and (5-10) that we have discussed in the previous section. System (1-4) has two equillibrium points, the disease free equillibrium point $E_{1}\left(H_{1}, 0, X_{1}, 0\right)$ where $H_{1}=K, X_{1}=\frac{(1-q) \tau}{c-b}$ with $q<1$ and $c>b$ and the endemic equilibrium point $E^{*}\left(H^{*}, S^{*}, X^{*}, Z^{*}\right)$ where $W^{*}=X^{*}+Z^{*}, H^{*}=\frac{r K}{r+k_{1} Z^{*}}$ that is, if the infected vector population increases then the healthy plant population decreases and vice versa, $S^{*}=\frac{r k_{1} K Z^{*}}{\left(r+k_{1} Z^{*}\right)\left(k_{3}+r\right)}$ that is, if the infected vector population increases then the infected plant population increases and $X^{*}=\frac{b Z^{*}+(1-q) \tau}{c+k_{2} S^{*}-b}$ with $c+k+2 S^{*}>b$. $Z^{*}$ is obtained from the quadratic equation

$$
A Z^{* 2}+B Z^{*}+C=0
$$

where

$A=k_{1} k_{3} c+r c k_{1}+r K k_{1} k_{2}>0$

$B=r k_{3} c+r^{2} c-r k_{1} k_{2} K W^{*}-q \tau k_{1}\left(k_{3}+r\right)>0$ if

$r k_{3} c+r^{2} c>r k_{1} k_{2} K W^{*}+q \tau k_{1}\left(k_{3}+r\right)$

$C=-\left(q \tau r k_{3}+q \tau r^{2}\right)<0$

Remark 1: Biologically, it means that in the absence of disease, the plant population approaches to the total plant density. But as disease affects the plant population then the healthy plant population decreases and the infected plant population increases to a very higher value. Similarly, the non-infected vector population decreases and the infected vector population increases.

Now, we will discuss the global stability of the interior equilibrium point $E^{*}$ by Lyapunov's direct method. This result has been established in the following theorem.

The jacobian corresponding to the system (1-4) is given as

$J=\left[\begin{array}{cccc}-r-k_{1} Z-\lambda & 0 & 0 & -k_{1} H \\ k_{1} Z & -\left(k_{3}+r\right)-\lambda & 0 & k_{1} H \\ 0 & -k_{2} X & b-c-k_{2} S-\lambda & b \\ 0 & k_{2} X & k_{2} S & -c-\lambda\end{array}\right]$

At the equilibrim point $E_{1}$ path is attracting in all the directions $H, S, X$, and $Z$ with the eigen values $-r,-\left(k_{3}+r\right)$, $-(c-b)$ and $-c$, respectively. The characteristics equation of the equation of the equillibrium point $E^{*}\left(H^{*}, S^{*}, X^{*}, Z^{*}\right)$ is

$$
\lambda^{4}+D \lambda^{3}+E \lambda^{2}+F \lambda+G=0
$$

where

$D=k_{3}+2 r+k_{2} S+k_{1} Z+2 c-b>0$

if $k_{3}+2 r+k_{2} S+k_{1} Z+2 c>b$

$E=\left(r+k_{1} Z\right)\left(r+k_{3}\right)+c\left(c+k_{2} S-b\right)-b k_{2} S-\left(b-2 c-k_{2} S\right)\left(k_{3}+2 r+k_{1} Z\right)$

if $\left(r+k_{1} Z\right)\left(r+k_{3}\right)+c\left(b-c-k_{2} S\right)>b k_{2} S+\left(b-2 c-k_{2} S\right)\left(k_{3}+2 r+k_{1} Z\right)$

$F=k_{1}^{2} k_{2} H Z X+k_{1} k_{2} H X+c\left(k_{3}+r\right)\left(c+k_{2} S-b\right)-b k_{2} S\left(k_{3}+r\right)+c\left(r+k_{1} Z\right)\left(c+k_{2} S-b\right)-b k_{2} S\left(r+k_{1} Z\right)-\left(r+k_{1} Z\right)\left(k_{3}+\right.$ $r)\left(b-2 c-k_{2} S\right)$

if $k_{1}^{2} k_{2} H Z X+k_{1} k_{2} H X+c\left(k_{3}+r\right)\left(b-c-k_{2} S\right)+c\left(r+k_{1} Z\right)\left(b-c-k_{2} S\right)>b k_{2} S\left(k_{3}+r\right)+b k_{2} S\left(r+k_{1} Z\right)+\left(r+k_{1} Z\right)\left(k_{3}+\right.$ $r)\left(b-2 c-k_{2} S\right)$

$G=c k_{1}^{2} k_{2} H Z X+c k_{1} k_{2} H X+c\left(k_{3}+r\right)\left(r+k_{1} Z\right)\left(c+k_{2} S-b\right)-b k_{2} S\left(r+k_{1} Z\right)\left(k_{3}+r\right)-b k_{1} k_{2} H X-b k_{1}^{2} k_{2} H Z X$

if $c k_{1}^{2} k_{2} H Z X+c k_{1} k_{2} H X+c\left(k_{3}+r\right)\left(r+k_{1} Z\right)\left(c+k_{2} S-b\right)>b k_{2} S\left(r+k_{1} Z\right)\left(k_{3}+r\right)+b k_{1} k_{2} H X+b k_{1}^{2} k_{2} H Z X$

Thus $E^{*}$ is locally asymptotically stable if $D>0, E>0, F>0, G>0$ and $D E-G>0$.

Theorem 1: $E^{*}$ is globally asymptotically stable if it satisfies the following conditions:

(i) $a_{12}^{2}<4 a_{11} a_{22}$, ii) $a_{34}^{2}<4 a_{33} a_{44}$ where $a_{11}=\left(r+k_{1} Z^{*}-\frac{K k_{1}}{2}\right), a_{12}=-A_{1} k_{1} Z^{*}, a_{34}=-\left(k_{2} A_{3} S^{*}+b A_{2}\right), a_{22}=$ $\left(A_{1}\left(k_{3}+r\right)-\frac{A_{1} K k_{1}}{2}-\frac{k_{2} A_{2} \tau}{2(c-b)}-\frac{k_{2} A_{3} \tau}{2(c-b)}\right), a_{33}=\left(k_{2} A_{2} S^{*}+c A_{2}-b A_{2}-\frac{k_{2} A_{2} \tau}{c-b}\right), a_{44}=\left(c A_{3}-\frac{K k_{1}}{2}-\frac{k_{2} A_{3} \tau}{2(c-b)}-\frac{A_{1} K k_{1}}{2}\right)$.

Proof: Let we consider the following positive definite function:

$$
V_{1}(H, S, X, Z)=\frac{1}{2} n_{1}^{2}+A_{1} \frac{1}{2} n_{2}^{2}+A_{2} \frac{1}{2} n_{3}^{2}+A_{3} \frac{1}{2} n_{4}^{2}
$$

then the time derivative of above equation is given as:

$\dot{V}_{1}(t)=n_{1} \dot{n_{1}}+A_{1} n_{2} \dot{n_{2}}+A_{2} n_{3} \dot{n_{3}}+A_{3} n_{4} \dot{n_{4}}$

where, $n_{1}=\left(H-H^{*}\right), n_{2}=\left(S-S^{*}\right), n_{3}=\left(X-X^{*}\right), n_{4}=\left(Z-Z^{*}\right)$

Now, from equations (1)-(4) and from equation (11) we get:

$\dot{V}_{1}(t)=\dot{V}_{11}+\dot{V}_{12}+\dot{V}_{13}+\dot{V}_{14}$ 
where

$\dot{V}_{11}=-\left\{\left(r+k_{1} Z^{*}-\frac{K k_{1}}{2}\right) n_{1}^{2}-\frac{r K k_{1}}{2} n_{4}^{2}\right\}$

$\dot{V}_{12}=-\left\{\left(A_{1}\left(k_{3}+r\right)-\frac{A_{1} K k_{1}}{2}\right) n_{2}^{2}-A_{1} k_{1} Z^{*} n_{1} n_{2}-\frac{A_{1} K k_{1}}{2} n_{4}^{2}\right\}$

$\dot{V}_{13}=-\left\{\left(k_{2} A_{2} S^{*}+c A_{2}-b A_{2}-\frac{k_{2} A_{2} \tau}{c-b}\right) n_{3}^{2}-\frac{k_{2} A_{2} \tau}{2(c-b)} n_{2}^{2}-b A_{2} n_{3} n_{4}\right\}$

$\dot{V}_{14}=-\left\{\left(c A_{3}-\frac{k_{2} A_{3} \tau}{2(c-b)}\right) n_{4}^{2}-\frac{k_{2} A_{3} \tau}{2(c-b)} n_{2}^{2}-k_{2} A_{3} S^{*} n_{3} n_{4}\right\}$

thus we can write equation (12) in the following form:

$\dot{V}_{1}=-\left\{a_{11} n_{1}^{2}+a_{12} n_{1} n_{2}+a_{22} n_{2}^{2}+a_{33} n_{3}^{2}+a_{44} n_{4}^{2}+a_{14} n_{1} n_{4}+a_{34} n_{3} n_{4}\right\}$

where, $a_{11}=\left(r+k_{1} Z^{*}-\frac{K k_{1}}{2}\right), a_{12}=-A_{1} k_{1} Z^{*}, a_{34}=-\left(k_{2} A_{3} S^{*}+b A_{2}\right), a_{22}=\left(A_{1}\left(k_{3}+r\right)-\frac{A_{1} K k_{1}}{2}-\frac{k_{2} A_{2} \tau}{2(c-b)}-\frac{k_{2} A_{3} \tau}{2(c-b)}\right)$, $a_{33}=\left(k_{2} A_{2} S^{*}+c A_{2}-b A_{2}-\frac{k_{2} A_{2} \tau}{c-b}\right), a_{44}=\left(c A_{3}-\frac{K k_{1}}{2}-\frac{k_{2} A_{3} \tau}{2(c-b)}-\frac{A_{1} K k_{1}}{2}\right)$. Now, we see that by Sylvester's criteria under the following conditions $\dot{V}_{1}(t)$ is negative definite.

$$
\text { (i) } a_{12}^{2}<4 a_{11} a_{22} \text {, ii) } a_{34}^{2}<4 a_{33} a_{44}
$$

Clearly, by Lyapunov's direct method $E^{*}$ is globally asymptotically stable. This completes the proof of the theorem.

Now we will discuss the stability of the system (5-10). Again for the system, we will analyze the disease-free equilibrium point affected by toxicant that is, $E_{1}\left(H_{1}, 0, X_{1}, 0, P, U\right)$ and the endemic equilirium point in the polluted environment that is $E^{*}\left(H^{*}, S^{*}, X^{*}, Z^{*}, P^{*}, U^{*}\right)$.

Now,consider the following system:

$\dot{x}(t)=f(t, x)$

$\dot{y}(t)=g(y)$

where, $f$ and $g$ are continuous and locally Lipschitz in $x$ in $R^{n}$, and solutions exists for all positive time. Equation (15) is called asymptotically autonomous with limit equation (14) if $f(t, x) \longrightarrow g(x)$ as $t \longrightarrow \infty$ uniformly for all $x$ in $R^{n}$.

Lemma: [15] Let $e$ be a locally asymptotically stable equilibrium of (15) and $\omega$ be the $\omega$-limit set of a forward bounded solution $x(t)$ of (14). If $\omega$ contains a point $y_{0}$ such that the solutions of (15), with $y(0)=y_{0}$ converges to $e$ as $t \longrightarrow \infty$, then $\omega=\{e\}$ i.e. $x(t) \longrightarrow e$ as $t \longrightarrow \infty$.

Corollary: If the solutions of the system (14) are bounded and the equilibrium $e$ of the limit system (15) is globally asymptotically stable then any solution $x(t)$ of the system (14) satisfies $x(t) \longrightarrow e$ as $t \longrightarrow \infty$.

The equation (9) and (10) can be solved explicitly and we obtain

$$
\limsup _{t \longrightarrow \infty} P(t) \leq P^{*}=\frac{Q}{h}
$$

and

$$
\limsup _{t \rightarrow \infty} U(t) \leq U^{*}=\frac{a_{1} P^{*}+\left(d_{1} \eta \phi / a_{1}\right)}{\left(l_{1}+l_{2}\right)}
$$

Thus, on applying above corollary in system (5)-(10) we get the following equivalent asymptotic autonomous system (see [16]):

$\frac{d H}{d t}=r(K-H)-k_{1} Z H-\alpha_{1} H U^{*}$

$\frac{d S}{d t}=k_{1} Z H-\left(k_{3}+r\right) S-\alpha_{2} S U^{*}$

$\frac{d X}{d t}=b(X+Z)-c X-k_{2} S X+(1-q) \tau$

$\frac{d Z}{d t}=k_{2} S X-c Z+q \tau$

Thus, it is clear that the asymptotic behaviour of the system (5)-(10) is equivalent to the asymptotic behaviour of the system (16)-(19), so that if the system (16)-(19) is stable then so is system (5)-(10). System (16)-(19) has also two equilibrium points, firstly disease-free equilibrium point in the presence of toxicant, that is, $E_{1}\left(H_{1}, S_{1}, X_{1}, Z_{1}\right)$ where $H_{1}=\frac{r K}{r+r_{1} U^{*}}$ and $X_{1}=$ $\frac{(1-q) \tau}{c-b}$ secondly, endemic equilibrium point in polluted environment, that is, $E^{*}\left(H^{*}, S^{*}, X^{*}, Z^{*}\right)$ where $H^{*}=\frac{r K}{r+k_{1} Z^{*}+\alpha_{1} U^{*}}$, $S^{*}=\frac{k_{1} Z^{*} H^{*}}{k_{3}+r+\alpha_{2} U^{*}}, X^{*}=\frac{b Z^{*}+(1-q) \tau}{c+k_{2} S^{*}-b}$ and $Z^{*}$ is obtained from the quadratic equation

$$
A_{2} Z^{* 2}+B_{2} Z^{*}+C_{2}=0
$$

where

$A_{2}=c k_{1}\left(k_{3}+r+\alpha_{2} U^{*}\right)+r K k_{1} k_{2}>0$ 
$B_{2}=\left(r c+c \alpha_{1} U^{*}-k_{1} q \tau\right)\left(k_{3}+r+\alpha_{2} U^{*}\right)-r K k_{1} k_{2} W^{*}>0$

if $r c+c \alpha_{1} U^{*}>k_{1} q \tau$ and

$\left(r c+c \alpha_{1} U^{*}-k_{1} q \tau\right)\left(k_{3}+r+\alpha_{2} U^{*}\right)>r K k_{1} k_{2} W^{*}$

$C_{2}=-\left(q \tau\left(k_{3}+r+\alpha_{2} U^{*}\right)\left(r+\alpha_{1} U^{*}\right)\right)<0$

Remark 2: Biologically, it means that that the disease free equilibrium point which was approaching to the total plant density earlier, now approaches to a lower level due to the effect of toxicant. But, it is not affecting the vector population as we haven't consider the effect of toxicant on the vector population. In the case of endemic equilibrium point, both the healthy and infected plant population will decline to a very lower level due to both the effect of disease and toxicant.

Now we will discuss the local stability analysis of the feasible boundary equilibrium point and and interior equilibrium point. The jacobian corresponding to the system(16)-(19) is :

$J=\left[\begin{array}{cccc}-r-k_{1} Z-\alpha_{1} U^{*}-\lambda & 0 & 0 & -k_{1} H \\ k_{1} Z & -\left(k_{3}+r\right)-\alpha_{2} U^{*}-\lambda & 0 & k_{1} H \\ 0 & -k_{2} X & b-c-k_{2} S-\lambda & b \\ 0 & k_{2} X & k_{2} S & -c-\lambda\end{array}\right]$

At the equilibrium point $E_{1}$ the jacobian is attracting in all the directions $H, S, X$ and $Z$ with eigen values $-\left(r+\alpha_{1} U^{*}\right)$, $-\left(k_{3}+r+\alpha_{2} U^{*}\right),-(c-b)$ and $-c$, respectively. The characteristic equation corresponding to the equilibrium point $E^{*}$ is given as

$$
\lambda^{4}+M \lambda^{3}+N \lambda^{2}+R \lambda+T=0
$$

where $M=k_{3}+2 r+k_{2} S+k_{1} Z+2 c+\alpha_{1} U^{*}+\alpha_{2} U^{*}-b>0$

if $k_{3}+2 r+k_{2} S+k_{1} Z+2 c \alpha_{1} U^{*}+\alpha_{2} U^{*}>b$

$N=\left(r+k_{1} Z+\alpha_{1} U^{*}\right)\left(r+k_{3}+\alpha_{2} U^{*}\right)+c\left(c+k_{2} S-b\right)-b k_{2} S-\left(k_{3}+r+\alpha_{2} U^{*}\right)\left(b-2 c-k_{2} S\right)-\left(r+k_{1} Z+\alpha_{1} U^{*}\right)\left(b-2 c-k_{2} S\right)$

if $\left(r+k_{1} Z+\alpha_{1} U^{*}\right)\left(r+k_{3}+\alpha_{2} U^{*}\right)+c\left(c+k_{2} S-b\right)>b k_{2} S+\left(k_{3}+r+\alpha_{2} U^{*}\right)\left(b-2 c-k_{2} S\right)+\left(r+k_{1} Z+\alpha_{1} U^{*}\right)\left(b-2 c-k_{2} S\right)$

$R=k_{1}^{2} k_{2} H Z X+k_{1} k_{2} H X+c\left(k_{3}+r+\alpha_{2} U^{*}\right)\left(c+k_{2} S-b\right)+c\left(r+k_{1} Z+\alpha_{1} U^{*}\right)\left(c+k_{2} S-b\right)-b k_{2} S\left(r+k_{1} Z+\alpha_{1} U^{*}\right)-$

$b k_{2} S\left(k_{3}+r+\alpha_{2} U^{*}\right)-\left(r+k_{1} Z+\alpha_{1} U^{*}\right)\left(k_{3}+r+\alpha_{2} U^{*}\right)\left(b-2 c-k_{2} S\right)$

if $k_{1}^{2} k_{2} H Z X+k_{1} k_{2} H X+c\left(k_{3}+r+\alpha_{2} U^{*}\right)\left(c+k_{2} S-b\right)+c\left(r+k_{1} Z+\alpha_{1} U^{*}\right)\left(c+k_{2} S-b\right)>b k_{2} S\left(r+k_{1} Z+\alpha_{1} U^{*}\right)+b k_{2} S\left(k_{3}+\right.$ $\left.r+\alpha_{2} U^{*}\right)+\left(r+k_{1} Z+\alpha_{1} U^{*}\right)\left(k_{3}+r+\alpha_{2} U^{*}\right)\left(b-2 c-k_{2} S\right)$

$T=c k_{1}^{2} k_{2} H Z X+c k_{1} k_{2} H X+c\left(k_{3}+r+\alpha_{1} U^{*}\right)\left(r+k_{1} Z+\alpha_{2} U^{*}\right)\left(c+k_{2} S-b\right)-b k_{2} S\left(r+k_{1} Z+\alpha_{1} U^{*}\right)\left(k_{3}+r+\alpha_{1} U^{*}\right)-$ $b k_{1} k_{2} H X-b k_{1}^{2} k_{2} H Z X$

if $c k_{1}^{2} k_{2} H Z X+c k_{1} k_{2} H X+c\left(k_{3}+r+\alpha_{1} U^{*}\right)\left(r+k_{1} Z+\alpha_{2} U^{*}\right)\left(c+k_{2} S-b\right)>b k_{2} S\left(r+k_{1} Z+\alpha_{1} U^{*}\right)\left(k_{3}+r+\alpha_{1} U^{*}\right)+$ $b k_{1} k_{2} H X+b k_{1}^{2} k_{2} H Z X$

Thus $E^{*}$ is locally asymptotically stable if $D>0, E>0, M>0, N>0$ and $M N-R>0$.

Now, we will discuss the global stability of the interior equilibrium point $E^{*}$ by Lyapunov's direct method. This result has been established in the following theorem.

Theorem 2: $E^{*}$ is globally asymptotically stable if it satisfies the following conditions

(i) $a_{12}^{2}<4 a_{11} a_{22}$, ii) $a_{34}^{2}<4 a_{33} a_{44}$ where $a_{11}=\left(r+k_{1} Z^{*}+\alpha_{1} U^{*}-\frac{K k_{1}}{2}\right), a_{12}=-A_{1} k_{1} Z^{*}, a_{34}=-\left(k_{2} A_{3} S^{*}+b A_{2}\right)$, $a_{22}=\left(A_{1} \alpha_{2} U^{*}+A_{1}\left(k_{3}+r\right)-\frac{A_{1} K k_{1}}{2}-\frac{k_{2} A_{2} \tau}{2(c-b)}-\frac{k_{2} A_{3} \tau}{2(c-b)}\right), a_{33}=\left(k_{2} A_{2} S^{*}+c A_{2}-b A_{2}-\frac{k_{2} A_{2} \tau}{c-b}\right), a_{44}=\left(c A_{3}-\frac{K k_{1}}{2}-\frac{k_{2} A_{3} \tau}{2(c-b)}-\frac{A_{1} K k_{1}}{2}\right)$. Proof: Let we consider the following positive definite function:

$V_{2}(H, S, X, Z)=\frac{1}{2} n_{1}^{2}+A_{1} \frac{1}{2} n_{2}^{2}+A_{2} \frac{1}{2} n_{3}^{2}+A_{3} \frac{1}{2} n_{4}^{2}$

then the time derivative of above equation is given as:

$\dot{V}_{2}(t)=n_{1} \dot{n_{1}}+A_{1} n_{2} \dot{n_{2}}+A_{2} n_{3} \dot{n_{3}}+A_{3} n_{4} \dot{n_{4}}$

where, $n_{1}=\left(H-H^{*}\right), n_{2}=\left(S-S^{*}\right), n_{3}=\left(X-X^{*}\right), n_{4}=\left(Z-Z^{*}\right)$

Now, from equations (16)-(19) and from equation (21) we get:

$\dot{V}_{2}(t)=\dot{V}_{21}+\dot{V}_{22}+\dot{V}_{23}+\dot{V}_{24}$

where

$\dot{V}_{21}=-\left\{\left(r+k_{1} Z^{*}-\frac{K k_{1}}{2}+\alpha_{1} U^{*}\right) n_{1}^{2}+\frac{K k_{1}}{2} n_{2}^{2}\right\}$

$\dot{V}_{22}=-\left\{A_{1} \alpha_{2} U^{*}+\left(A_{1}\left(k_{3}+r\right)-\frac{A_{1} r K k_{1}}{2}\right) n_{2}^{2}-A_{1} k_{1} Z^{*} n_{1} n_{2}-\frac{A_{1} r K k_{1}}{2} n_{4}^{2}\right\}$

$\dot{V}_{23}=-\left\{\left(k_{2} A_{2} S^{*}+c A_{2}-b A_{2}-\frac{k_{2} A_{2}^{2} \tau}{c-b}\right) n_{3}^{2}-\frac{k_{2} A_{2} \tau}{2(c-b)} n_{2}^{2}-b A^{2} n_{3} n_{4}\right\}$

$\dot{V}_{24}=-\left\{\left(c A_{3}-\frac{k_{2} A_{3} \tau}{2(c-b)}\right) n_{4}^{2}-\frac{k_{2} A_{3} \tau}{2(c-b)} n_{2}^{2}-k_{2} A_{3} S^{*} n_{3} n_{4}\right\}$

thus we can write equation (22) in the following form:

$\dot{V}_{2}=-\left\{a_{11} n_{1}^{2}+a_{12} n_{1} n_{2}+a_{22} n_{2}^{2}+a_{33} n_{3}^{2}+a_{44} n_{4}^{2}+a_{14} n_{1} n_{4}+a_{34} n_{3} n_{4}\right\}$

where, $a_{11}=\left(r+k_{1} Z^{*}+\alpha_{1} U^{*}-\frac{K k_{1}}{2}\right), a_{12}=-A_{1} k_{1} Z^{*}, a_{34}=-\left(k_{2} A_{3} S^{*}+b A_{2}\right), a_{22}=\left(A_{1} \alpha_{2} U^{*}+A_{1}\left(k_{3}+r\right)-\frac{A_{1} K k_{1}}{2}-\right.$ $\left.\frac{k_{2} A_{2} \tau}{2(c-b)}-\frac{k_{2} A_{3} \tau}{2(c-b)}\right), a_{33}=\left(k_{2} A_{2} S^{*}+c A_{2}-b A_{2}-\frac{k_{2} A_{2} \tau}{c-b}\right), a_{44}=\left(c A_{3}-\frac{K k_{1}}{2}-\frac{k_{2} A_{3} \tau}{2(c-b)}-\frac{A_{1} K k_{1}}{2}\right)$.

Now, we see that by Sylvester's criteria under the following conditions $\dot{V}_{2}(t)$ is negative definite. 


$$
\text { (i) } a_{12}^{2}<4 a_{11} a_{22} \text {, ii) } a_{34}^{2}<4 a_{33} a_{44}
$$

Clearly, by Lyapunov's direct method $E^{*}$ is globally asymptotically stable.

In the next section we will show the stability of both the models numerically by using MATLAB software. This completes the proof of the theorem.

\section{Numerical example}

We consider the following set of parameters for the model 1( $a$ )

$r=0.2, b=0.1, c=0.2, K=500, k_{1}=k_{2}=0.05, k_{3}=0.04, q=0.024, \tau=0.169, A_{1}=0.001, A_{2}=2, A_{3}=100$

Then we get the following results:

(1) Region of attraction for the Model 1(a) is:

$$
R_{1}=\left\{(H, S, X, Z) \in R_{+}^{4}: 0 \leq H+S \leq 500,0 \leq X+Z \leq 1.69\right\}
$$

and the equilibrium points are :

(i) Disease free equilibrium point $E_{1}\left(H_{1}, 0, X_{1}, 0\right)$ where $H_{1}=500$, and $X_{1}=1.6490$

(ii) Endemic Equilibrium point $E^{*}\left(H^{*}, S^{*}, X^{*}, Z^{*}\right)$ where $H^{*}=354.8616, S^{*}=121.0429, X^{*}=0.0534$ and $Z^{*}=1.6365$.

The local stability of the system by the jacobian matrix gives the following eigen values :

$\lambda_{1}=-6.2602, \lambda_{2}=-0.2569+.0058 i, \lambda_{3}=-.2569-.0058 i, \lambda_{4}=-0.1$

Since all the eigen values are negative, thus the system is locally asymptotically stable and is justified numerically for the above set of parameters. The conditions of the global stability analysis, is are also satisfied for the above parameters which ensures the global stability of the system numerically.

Now, we consider the following set of parameters for model $1(b)$ :

$\alpha_{1}=0.01, \alpha_{2}=0.02, Q=5, a_{1}=0.1, d_{1}=0.5, \theta=0.01, \beta=0.1, l_{1}=0.1, l_{2}=0.1$

and the rest of the parameters have same numerical value as given in Model $1(a)$. Then we get the following results:

(1) Region of attraction for he Model 1(b) is:

$$
R_{2}=\left\{(H, S, X, Z) \in R_{+}^{4}: 0 \leq H+S \leq 500,0 \leq X+Z \leq 1.69,0 \leq P \leq 500,0 \leq U \leq 250.025\right\}
$$

and the equilibrium points are :

(i) Disease free equilibrium point $E_{1}\left(H_{1}, 0, X_{1}, 0\right)$ where $H_{1}=37.0329$, and $X_{1}=1.6490$

(ii) Endemic Equilibrium point $E^{*}\left(H^{*}, S^{*}, X^{*}, Z^{*}\right)$ where $H^{*}=37.0178, S^{*}=.0084, X^{*}=1.6663$ and $Z^{*}=.0238$.

The local stability of the system by the jacobian matrix gives the following eigen values :

$\lambda_{1}=-5.2709, \lambda_{2}=-2.7014, \lambda_{3}=-0.1701, \lambda_{4}=-0.1$

Thus the system is locally asymptotically stable is also justified numerically for the above set of parameters. The conditions of the global stability analysis, are also satisfied for the above parameters which ensures the global stability of the system numerically.

Remark 3: Biologically, the equilibria level of the healthy plant population decreases as the disease effects the plant population and the infected plant population increases. But as the toxicant effects both the healthy and the infected plants both, then the equilibria level of the total plant density declines to a very lower level.

\section{Discussion}

In this paper, two models have been discussed. In the first model 1(a), the effect of disease on a plant population via vector population has been studied. It has been observed that the plant population, decreases to a lower level as the disease effects it, but the system remains stable. In the second model 1(b), the effect of both disease and toxicant has been studied on the plant population and it has been concluded that the plant population declines to a very lower level due to the simultaneous effect of both disease and toxicant. The local and global stability conditions of both the models have been derived and finally numerical justification in support of our results have been also shown. 


\section{References}

[1] Hallam, T.G., Clark, C.E., Lassiter, R.R : Effects of toxicant on populations: a qualitative approach I. Equillibrium environmental expose. Ecol. Modelling 18, 291-304 (1983).

[2] Hallam, T.G., Clark, C.E., Jordan, G.S : Effects of toxicant on populations: a qualitative approach II. First order Kinetics. J. MAth. Biol. 18, 25-37 (1983).

[3] Hallam, T.G., De Luna, J.T : Effects of toxicant on populations: a qualitative approach III. Environmental and food chain pathways. J. Theor. Biol. 109, 411-429 (1984).

[4] Hallam, T.G., MA Zhien : Persistence in population models with demographic fluctuations. J. math. Biol. 24, 327-339 (1986).

[5] De Luna, J.T., Hallam T.G : Effects of toxicant on populations: a qualitative approach III. resource-consumer-Toxicant models. Ecol. Modelling 35, 249-273 (1987).

[6] Debasis Mukherjee, Persistence and global stability of a population in a polluted environment with delay, journal of biological Systems. 10, 225-232 (2002).

[7] Zhien Ma, G.Cui, W.Wang, Persistence and extinction of a population in a polluted environment, math. Biosci. 101, 75 (1990).

[8] Camphell C.L, Madden LV, Introduction to plant Disease Epidemiology, New York, USA: Wiley, (1990).

[9] Hughes G, Madden LV, Aggregation and incidence of disease. Plant Pathology 41, 657-60, (1992).

[10] Hughes G, Madden LV, Using the beta-binomial distribution to describe aggregated patterns of disease incidence. Phytopathology, 83, 759-63 (1993).

[11] Hughes G, Madden LV, Comment- Using spatial and temporal patterns of Armillaria root disease to formulate management recommendations for Ontario's black spruce(Picea mariana) seed orchard. Canasian Journal of Forest Research 28, 153-8 (1998).

[12] Madden LV, Hughes G, Plant disease incidence : distributions, heterogeneity, and temporal analysis. Annual Review of Phytopathology 33, 529-64 (1995).

[13] Madden LV, Nault LR, Murral DJ, Apelt MR, Spatial Pattern Analysis of the incidence of aster yellows disease in lettuce. Researches on population Ecology 37, 279-89 (1995).

[14] Madden LV, Pirone TP, Raccah B, Analysis of spatial patterns of virus-induced tobacco plants. Phytopathology 77, 1409-17 (1987).

[15] H.R. Thieme, 1992, Convergence results and a Poincare-Bendixon trichotomy for asymptotically autonomous differential equations. J. Math. Biol., 30, pp. 755-763.

[16] L. Han, Z. Ma, H.W. Hethcote, 2001, Four predator-prey models with infectious diseases. Math. Comp. Modelling, 34, pp. 849-858. 